\title{
Francesco Collamati, supervised by Riccardo Faccini: An intraoperative beta-probe for cancer surgery, Springer Theses
}

\author{
Springer International Publishing Switzerland 2016, ISBN 978-3-319-33698-5
}

\author{
Gianluca De Matteis $^{1} \cdot$ Luigi Mansi ${ }^{2}$
}

Received: 22 September 2017 / Accepted: 9 October 2017 /Published online: 2 November 2017

(C) Springer-Verlag GmbH Germany 2017

The book, organized into six chapters for a total of about 100 pages, is derived from a $\mathrm{PhD}$ thesis concerning a very important emerging field of nuclear medicine, radio-guided surgery (RGS). The author is Francesco Collamati who produced this text having as Supervisor Prof. Riccardo Faccini, from the Department of Physics at the University La Sapienza of Rome, Italy.

The book summarizes a variety of topics and fields: tumor biology (cutaneous melanoma, breast cancer, brain tumors, and NET); physical characteristics of used radiations ( $\gamma$ radiation in protocols already in use in clinical practice, but also $\beta^{+}$and $\beta^{-}$less codified in practice to date); probe's structure and function analysis of three prototypes; statistical analysis of results with explanation through mathematical models (the FLUKA Monte Carlo code).

A great interest of the book is derived from the creation of a thread built on mathematical models and simulations. In par-

Luigi Mansi

mansi.luigi@libero.it

1 Università Federico II, Naples, Italy

2 Centro Interuniversitario per lo Sviluppo Sostenibile (CIRPS), Naples, Italy ticular, in chapter 6 , the author theoretically discusses radiodosimetry in the personnel involved in radio-guided surgery procedures using a mathematical model mixing the FLUKA Monte Carlo code with DICOM PET data. The importance of this approach is that there are no data in the literature on radiation exposure in the use of $\beta^{+}$emitting radiopharmaceuticals. Therefore, this theoretical report also represents a feasibility study, supporting the development of commercial tools based on the detection of $\beta^{+}$and $\beta^{-}$radiation.

The innovations discussed are so important that 1 year after the completion of this work, the technique, until then only theorized on mathematical models, was applied for the first time on a patient with meningioma. A paper on this experience has been published in the Journal of Nuclear Medicine and Molecular Imaging.

Therefore, in our opinion, the book can be interesting for all the people interested in radio-guided surgery, including not only physicians but also physicists, mathematicians, and technologists. 